\title{
A subset of colorectal cancers with cross-sensitivity to olaparib and oxaliplatin
}

Sabrina Arena ${ }^{1,2}$, Giorgio Corti ${ }^{1}$, Erika Durinikova ${ }^{1}$, Monica Montone ${ }^{1}$, Nicole M. Reilly ${ }^{3}$, Mariangela Russo ${ }^{1,2}$, Annalisa Lorenzato ${ }^{1,2}$, Pamela Arcella ${ }^{1,2}$, Luca Lazzari ${ }^{4}$, Giuseppe Rospo $^{1}$, Massimiliano Pagani ${ }^{5,6}$, Carlotta Cancelliere ${ }^{1}$, Carola Negrino ${ }^{1,2}$, Claudio Isella ${ }^{1}$, Alice Bartolini ${ }^{1}$, Andrea Cassingena ${ }^{7}$, Alessio Amatu $^{7}$, Gianluca Mauri ${ }^{7,8}$, Andrea SartoreBianchi $^{7,8}$, Gloria Mittica ${ }^{1}$, Enzo Medico ${ }^{1,2}$, Silvia Marsoni ${ }^{4,7}$, Michael Linnebacher ${ }^{9}$, Sergio Abrignani $^{5,10}$, Salvatore Siena ${ }^{7,8}$, Federica Di Nicolantonio ${ }^{1,2}$ and Alberto Bardelli ${ }^{1,2}$

${ }^{1}$ Candiolo Cancer Institute, FPO - IRCCS, Candiolo (TO), Italy; ${ }^{2}$ Department of Oncology, University of Torino, Candiolo (TO), Italy; ${ }^{3}$ Fondazione Piemontese per la Ricerca sul Cancro ONLUS, Candiolo (TO), Italy; ${ }^{4}$ IFOM, The FIRC Institute of Molecular Oncology, Milan, Italy; ${ }^{I}$ NGM, Istituto Nazionale Genetica Molecolare "Romeo ed Enrica Invernizzi", Milan, Italy; ${ }^{6}$ Department of Medical Biotechnology and Translational Medicine, Università degli Studi di Milano, Milano, Italy; ${ }^{7}$ Niguarda Cancer Center, Grande Ospedale Metropolitano Niguarda, Milan, Italy; ${ }^{8}$ Department of Oncology and Hemato-Oncology, Università degli Studi di Milano, Milan, Italy; ${ }^{9}$ Department of General Surgery, Molecular Oncology and Immunotherapy, University of Rostock, Rostock, Germany; ${ }^{10}$ Department of Clinical Sciences and Community Health, Università degli Studi di Milano, Milano, Italy.

Corresponding authors: Prof. Alberto Bardelli, Department of Oncology, University of Torino, SP 142 km 3.95, 10060 Candiolo (TO), Italy. Tel: +39-011-9933235. E-mail: alberto.bardelli@unito.it. Prof. Federica Di Nicolantonio, Department of Oncology, University of Torino, SP 142 km 3.95, 10060 Candiolo (TO), Italy. Tel: +39-011-9933827. E-mail: Federica.dinicolantonio@unito.it.

Running Title: Olaparib and oxaliplatin cross-sensitivity in CRC

Keywords: colorectal cancer; Olaparib; PARPi; oxaliplatin; organoids; homologous recombination

\section{Additional Information:}

Financial support: The research leading to these results has received funding from Fondazione AIRC under 5 per Mille 2018 - ID. 21091 program - P.I. Bardelli Alberto, G.L. Siena Salvatore, G.L. Medico Enzo, G.L. Abrignani Sergio, G.L. Marsoni Silvia, G.L. Di Nicolantonio Federica. The research leading to these results has received funding from AIRC under MFAG 2017 -ID 20236 
project -P.I. Arena Sabrina. This work was also supported by European Community's Seventh Framework Programme under grant agreement no. 602901 MErCuRIC (A.B.); H2020 grant agreement no. 635342-2 MoTriColor (A.B. and S.S.); IMI contract n. 115749 CANCER-ID (A.B.); AIRC IG 2018 - ID. 21923 project - PI Bardelli Alberto; AIRC IG n. 17707 and IG n. 21407 projects - PI Di Nicolantonio Federica; AIRC IG n. 20685 (S.S.); Terapia Molecolare Tumori by Fondazione Oncologia Niguarda Onlus (A.S.B. and S.S.); Genomic-Based Triage for Target Therapy in Colorectal Cancer Ministero della Salute, Project n. NET 02352137 (A.S.B., A.B., F.D.N. and S.S.). TRANSCAN-2 JTC 2014 contract no. TRS-2015-00000060 INTRACOLOR (S.A.); AIRC-CRUK-FC AECC Accelerator Award contract 22795 (A.B.); Fondazione Piemontese per la Ricerca sul Cancro-ONLUS 5 per mille 2014 e 2015 Ministero della Salute (A.B. and F.D.N.); Ministero Salute, RC 2019 (A.B. and F.D.N.).

Conflicts of Interest: A.Bard. is an advisory board member for Roche, Biocartis, Third Rock, Neophore, Phoremost, Illumina and Horizon Discovery; A.S.B. is an advisory board member for Amgen, Bayer and Sanofi; S.S. is an advisory board member for Clovis; A.A. is an advisory board member for Bayer, Roche and Amgen. The other authors declare no potential conflicts of interest.

\section{Abstract}

Purpose: Defects in the homologous recombination $(\mathrm{HR})$ repair pathway are of clinical interest due to sensitivity of HR-deficient cells to poly (ADP-ribose) polymerase (PARP) inhibitors. We were interested in defining PARP vulnerability in patients with metastatic colorectal cancer $(\mathrm{mCRC})$ carrying KRAS and BRAF mutations that display poor prognosis, have limited therapeutic options and represent an unmet clinical need. Experimental Design: We tested $C R C$ cell lines, patient-derived organoids (PDOs) and patient-derived xenografts (PDXs) enriched for KRAS and BRAF mutations for sensitivity to the PARP inhibitor olaparib, and the chemotherapeutic agents oxaliplatin and 5fluorouracil (5-FU). Genomic profiles and DNA repair proficiency of CRC models were compared to pharmacological response. Results: Thirteen out of ninety-nine (around 13\%) CRC lines were highly sensitive to clinically active concentrations of olaparib and displayed functional deficiency in HR. Response to PARP blockade was positively correlated with sensitivity to oxaliplatin in CRC cell lines as well as patient-derived organoids. Treatment of PDXs with olaparib impaired tumor growth and maintenance therapy with PARP blockade after initial oxaliplatin response delayed disease progression in mice. Conclusions: These results indicate that a CRC subset characterized by poor prognosis and limited therapeutic options is vulnerable to PARP inhibition and suggest 
that PDO-based drug-screening assays can be used to identify CRC patients likely to benefit from olaparib. As $\mathrm{mCRC}$ patients almost invariably receive therapies based on oxaliplatin, 'maintenance' treatment with PARP inhibitors warrants further clinical investigation.

\section{Translational Relevance (150 words)}

There is a need to expand therapeutic options for a significant subset of $\mathrm{mCRC}$ patients who do not benefit from targeted or immune therapies. Through a comprehensive screening of a large collection of CRC cell lines enriched for RAS/BRAF mutations, we found that up to $13 \%$ cases are sensitive to the poly (ADP-ribose) polymerase (PARP) inhibitor olaparib. We detected pharmacological cross-sensitivity between olaparib and oxaliplatin, which is of immediate translational relevance. Maintenance therapy with PARP blockade after initial oxaliplatin response was effective in delaying disease progression in CRC patient-derived xenografts. This study lay a rationale for the design of trials testing a maintenance therapy with PARP inhibitors in the subset of mCRCs patients with complete or partial response to prior oxaliplatin treatment.

Key words: colorectal cancer, PARPi, Olaparib, Oxaliplatin, homologous recombination

\section{INTRODUCTION}

Treatment of metastatic colorectal cancer (mCRC) has improved over the last fifteen years since the introduction of EGFR targeted therapy, antiangiogenic agents and the use of intensive triplet chemotherapy regimens based on fluoropyrimidines, oxaliplatin and irinotecan (1). Immune checkpoint inhibitors have been shown to induce durable responses in a subset of approximately $5 \% \mathrm{mCRC}$ patients that carry defective mismatch repair (MMRd) or are microsatellite unstable (MSI) $(2,3)$. Targeted therapy trials are ongoing with promising results in subsets of molecularly selected CRCs, such as BRAF mutant or HER2 amplified cases $(4,5)$ and these targeted combinations are expected to enter clinical practice in a short timeframe. However, median overall survival in mCRC 
patients has reached a plateau that ranges from 18 months, in cases of RAS mutant and right colonic tumors, to 42 months in BRAF/RAS wild-type and left side cancers (6-8). In summary, while the overall survival of $\mathrm{mCRC}$ patients has been increased by combining and fine-tuning the use of cytotoxics, targeted agents and immunotherapy, the impact of these advances has been incremental rather than transformative.

Accordingly, there is a need to improve disease control in mCRC patients and prolong overall survival, particularly for patients who are not eligible for targeted agents or immune therapy. Furthermore, intrinsic and acquired drug resistance, as well as the neuropathy associated with oxaliplatin-containing regimens (9), limits the duration of long-term disease control and represent a clinical problem that needs to be addressed.

Molecular profiling of large CRC datasets has revealed subsets of cases with defects in DNA repair pathways, some of which may be amenable to therapeutic targeting. Germline pathogenic variants of $B R C A 1$, associated with defects in the homologous recombination $(\mathrm{HR})$ repair pathway, are emerging as a risk factor for CRC (10), particularly for early onset CRC (11). Importantly, recent studies have reported that up to $15 \%$ of individuals carry germline or somatic genetic defects in HR repair genes (12-14). In other tumor types such as breast and ovarian cancers, defects in the HR repair machinery, due to alterations in BRCA or other genes, such as RAD51 and PALB2, confers the so-called 'BRCAness' phenotype (15). These tumors often display sensitivity to specific DNA damaging agents including platinum compounds and poly (ADP-ribose) polymerase (PARP) inhibitors (1618).

Four PARP inhibitors are approved in slightly different clinical niches for specific malignancies carrying germline BRCA mutations, in previously treated ovarian and breast cancer patients. Of these, only one is one also approved for maintenance therapy in ovarian, fallopian tube and primary peritoneal cancer patients in remission after platinumbased therapy (18-29). Approval for use in germ-line BRCA mutant prostate and pancreatic cancers is expected in 2020. Data in other malignancies are either immature or controversial. The development of PARP inhibitors in CRC, alone or in combination with other cytotoxic agents, has been hampered by toxicity or the lack of patient selection (30-33). On the other hand, preclinical testing of PARP inhibitors in molecularly annotated 
bowel tumors has not been performed extensively. Hence, in this work we set to establish the prevalence of exquisite sensitivity to PARP inhibition in a large collection of CRC cell lines and preclinical models, with the ultimate goal of defining potential predictive markers for guiding clinical development of PARP inhibitors in bowel cancer. We decided to focus on MMR proficient KRAS and BRAF mutant CRC, as these tumors lack effective treatments or, despite novel combined target therapies, still display dismal outcome. Our work revealed that response to olaparib in CRC cell lines was positively correlated with sensitivity to oxaliplatin treatment in vitro. While olaparib sensitivity could not be pinpointed by genomic defects in BRCA or other HR repair genes, we found that functional assays based on detection of DNA damage response were able to predict vulnerability to PARP inhibition in cell lines and patient-derived models.

\section{MATERIALS AND METHODS}

\section{Cell lines and cell authentication}

All CRC cell lines are part of datasets we previously characterized $(34,35)$. Each cell line was cultured in its specific media and conditions. All cell lines were grown at $37^{\circ} \mathrm{C}$ in a $5 \% \mathrm{CO}_{2}$ air incubator. Cell lines were routinely checked for mycoplasma contamination using the Venor GeM Classic Kit (Minerva Biolabs) according to the manufacturer's protocol. The genetic identity of cell lines was performed using the PowerPlex® $16 \mathrm{HS}$ System (Promega), through Short Tandem Repeats (STR) at 16 different loci (D5S818, D13S317, D7S820, D16S539, D21S11, vWA, TH01, TPOX, CSF1PO, D18S51. D3S1358, D8S1179, FGA, Penta D, Penta E, and amelogenin). Amplicons from multiplex PCRs were separated by capillary electrophoresis (3730 DNA Analyzer, Applied Biosystems) and analyzed using GeneMapperID v.3.7 software (Life Technologies).

\section{PDX and organoid establishment}

Tumor samples were obtained from patients treated at Niguarda Cancer Center (Milano, Italy). All patients provided informed written consent, samples were procured and the 
study was conducted in accordance with the Declaration of Helsinki and under the approval of the local Independent Ethical Committee (protocol 194/2010). To generate patient-derived xenografts (PDXs), tumor specimens were subcutaneously implanted in 7-week-old NOD-SCID mice (Charles River Laboratory). All animal procedures were approved by the Ethical Committee of the Candiolo Cancer Institute and by the Italian Ministry of Health.

Patient \#1 (case HROC278 (35)) and patient \#3 organoids were established at Candiolo Cancer Center from PDX models obtained following procedures described below. To generate organoids, PDX tissue was dissociated into single-cell suspension by mechanical dissociation using the Gentle MACS Dissociator (Miltenyi Biotec) and enzymatic degradation of the extracellular matrix using the Human Tumor Dissociation Kit (Miltenyi Biotec) according to the manufacturer's protocol. Cell suspension was centrifuged at $400 \mathrm{~g}$ for 5 minutes and then the pellet was resuspended with organoid basal medium (consisting of advanced DMEM/F12 medium containing $100 \mathrm{U} / \mathrm{mL}$ penicillin, $100 \mu \mathrm{g} / \mathrm{mL}$ streptomycin, $2 \mathrm{mM}$ GlutaMAX and $10 \mathrm{mM}$ Hepes). Final cell

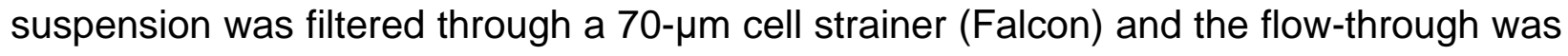
centrifuged three times. At the end of the washing phase, the pellet was resuspended in Matrigel (Corning) and $50 \mu \mathrm{L}$ of organoids-Matrigel suspension were dispensed into the center of each well of a $37^{\circ} \mathrm{C}$ pre-warmed 24 -well plate. Different densities of tumor cells were plated and left to solidify for $10-30 \mathrm{~min}$ at $37^{\circ} \mathrm{C}$ before ENAS or WENRAS medium was added and the cells were incubated at $37^{\circ} \mathrm{C}, 5 \% \mathrm{CO}_{2}$. To prepare ENAS medium, the following reagents were added to Basal medium: $1 \times$ B27 supplement (Invitrogen), $1 \times$ N2 supplement (Invitrogen), $1.25 \mathrm{mM} \mathrm{N}$-acetyl-cysteine (Sigma Aldrich), $10 \mathrm{mM}$ nicotinamide (Sigma Aldrich), $10 \mathrm{nM}$ gastrin (Sigma), $50 \mathrm{ng} / \mathrm{mL}$ human EGF (Life Technologies), $100 \mathrm{ng} / \mathrm{mL}$ Noggin (PeproTech), $500 \mathrm{nM}$ TGFb type I receptor inhibitor A83-01 (Tocris) and $10 \mu \mathrm{M}$ p38 MAPK inhibitor SB202190 (Sigma Aldrich). During the first three days of organoid establishment, ROCK inhibitor (10 $\mu \mathrm{M}$, Selleckchem) and GSK inhibitor (3 $\mu \mathrm{M}$, Selleckchem) were added. WENRAS medium was prepared by adding 10\% RSPO1-CM (conditioned medium) and 50\% WNT3a-CM to ENAS medium. 
Patient \#2, patient \#4 and patient \#5 organoids were established directly from tissue biopsy obtained at the time of surgery. Organoids from patient\#2 were established at INGM (Istituto Nazionale Genetica Molecolare "Romeo ed Enrica Invernizzi", Milan), while organoids from patients \#4 and \#5 were established at Candiolo Cancer Institute. Briefly, tumor tissue was first smashed in small pieces and incubated in PBS with collagenase A $(0.5 \mathrm{mg} / \mathrm{mL}$; Roche), hyaluronidase (20 $\mathrm{gg} / \mathrm{ml}$; Sigma) and $10 \mu \mathrm{M}$ ROCK inhibitor for 30 min at $37{ }^{\circ} \mathrm{C}$ with shaking. After incubation, 5\% FBS was added and the mixture was centrifuged at $400 \mathrm{~g}$ for 5 minutes. The pellet was washed twice in PBS to remove debris and collagenase. At the end of the washing phase, the pellet was resuspended in Matrigel (Corning) and $50 \mu \mathrm{L}$ of organoids-Matrigel suspension were dispensed into the center of each well of a $37^{\circ} \mathrm{C}$ pre-warmed 24-well plate. Different densities of tumor cells were plated and left to solidify for $10-30 \mathrm{~min}$ at $37^{\circ} \mathrm{C}$ before ENAS medium was added and the cells were incubated at $37{ }^{\circ} \mathrm{C}, 5 \% \mathrm{CO}_{2}$. Fresh medium was replaced every $2-3$ days. Outgrowing organoids were passaged every 10-15 days after mechanical and enzymatic disruption. Organoid drug treatment was performed in each organoid-specific culture medium at $37^{\circ} \mathrm{C}$ and $5 \% \mathrm{CO}_{2}$.

\section{Genomic DNA extraction, exome sequencing and bioinformatic analysis}

Genomic DNA samples were extracted from each cell line using ReliaPrep gDNA Tissue Miniprep System (Promega) and sent to IntegraGen (France) for exome sequencing. Data analysis was performed at the Candiolo Cancer Institute following procedures as previously described (36). All synonymous variants were filtered out and only SNVs with VAF $>10 \%$ were subsequently annotated by SIFT and PolyPhen algorithms.

\section{MSS/ MSI analysis}

The microsatellite instability (MSI) status was evaluated by using the MSI Analysis System kit (Promega) according to manufacturer's protocol. The analysis requires a multiplex amplification of seven markers including five mononucleotide repeat markers (BAT-25, BAT-26, NR-21, NR-24 and MONO-27) and two pentanucleotide repeat markers (Penta $C$ and Penta $D$ ). The products were analyzed by capillary electrophoresis in a single injection using $\mathrm{ABI} 3730$ DNA Analyzer capillary electrophoresis system 
(Applied Biosystems). The results were analyzed using GeneMapper V5.0 software. Samples with instability in two or more markers were defined as MS-instable (MSI-H). Samples with no detectable alterations were defined as MS-stable (MSS).

\section{Analysis of Mutational signatures}

Mutational signatures were calculated selecting the nucleotide changes of variants from the small nucleotide variants (SNVs) report file(36): variants present in common dbSNP version 150 or in a panel of 40 normal samples previously sequenced were excluded, and only SNVs showing a statistical significant $p$ value (less than 0.05 calculated with a binomial test on allele count and depth), as well an allelic frequency greater or equal to $10 \%$ were selected. These variants were passed through the web application "Mutational Signatures in Cancer" (MuSiCa)(37). Signature profiles were calculated using the six substitution subtypes: $C>A, C>G, C>T, T>A, T>C$, and $T>G$ (referring to the pyrimidine of the mutated Watson-Crick base pair). Information on $5^{\prime}$ and $3^{\prime}$ base context to each mutated nucleotide was incorporated to generate 96 possible mutation types. The output file of MuSiCa including the contribution values of 30 signatures (38) was used to create the heatmap.

\section{Drugs}

Olaparib (AZD2281, Ku-0059436, S1060), niraparib (S2741), rucaparib (S1098), oxaliplatin (S1224) and 5-fluorouracil (S1209) were purchased from Selleck Biochem.

\section{Oxaliplatin and 5-fluorouracil testing}

Three thousands cells/well were seeded in $100 \mu \mathrm{l}$ complete growth medium in 96-well plastic culture plates at day 0 . The following day, serial dilutions $(100 \mu l)$ of oxaliplatin $(0-$ $12.5 \mu \mathrm{M})$ or 5 -FU $(0-20 \mu \mathrm{M})$ were added. Plates were incubated at $37^{\circ} \mathrm{C}$ in $5 \% \mathrm{CO}_{2}$ for 6 days, after which cell viability was assessed by measuring ATP content through Cell TiterGlo Luminescent Cell Viability assay (Promega). Luminescence was measured by the SPARK M10 (Tecan) plate reader. Treated wells were normalized to untreated wells.

\section{Olaparib testing}


Olaparib sensitivity was tested in long-term proliferation assays. Briefly, cells were seeded in 24-wells plates at day 0 and the following day they were treated with serial dilutions of olaparib $(0.5-15 \mu \mathrm{M})$. The treatment was refreshed every week and the assay was stopped when untreated cells reached confluency (from 10 days to 2 weeks of treatment). Plates were fixed with 4\% paraformaldehyde (Santa Cruz) and stained with $1 \%$ crystal violet-methanol solution (Sigma-Aldrich), which was then solubilized by $10 \%$ acetic acid and quantified by measuring the absorbance at $600 \mathrm{~nm}$ at the SPARK M10 (Tecan) plate reader. Treated wells were normalized to untreated wells.

\section{Immunofluorescence detection of RAD51 foci}

Cells were grown on glass coverslips $\left(2 \times 10^{5}\right.$ cells per well) in a 6 -well plate. Twenty-four hours after plating, the cells were exposed to ionizing radiation at the indicated doses and allowed to recover for 4 hours. After recovery, the cells were fixed in $4 \%$ paraformaldehyde for $20 \mathrm{~min}$ at room temperature and permeabilized with $0.1 \%$ TritonX100 in PBS for 5 minutes. Cells were incubated at room temperature with 1\% BSA in PBS for 30 minutes, followed by incubation overnight at $4{ }^{\circ} \mathrm{C}$ with the following primary antibodies diluted in PBS containing $1 \%$ of BSA and $1 \%$ of donkey serum: anti-phosphoHistone H2AX (Ser139) rabbit monoclonal antibody (Bethyl Laboratories) (1:600) and anti-RAD51 rabbit polyclonal antibody (Millipore) (1:100). After washing, cells were fluorescently labeled according to the primary antibody used with an Alexa Fluor® 555 donkey anti-mouse antibody or Alexa Fluorß 488 donkey anti-rabbit antibody (Molecular Probes, Eugene, USA) (1:400) for 1 hour at room temperature. Nuclei were stained with DAPI. A Leica DMI6000B fluorescence microscope (Leica Microsystems, Wetzlar, Germany) under a 40X dry objective was used to detect $\mathrm{Y}-\mathrm{H} 2 \mathrm{AX}$ and RAD51 foci. Images were captured at 10 individual z-planes and were merged using the " $Z$ Project" function in ImageJ. Individual nuclei were scored for foci positivity as identified based upon signal intensity above general background staining levels and present within the nucleus as assessed by DAPI staining. Cells containing $\geq 5$ distinct foci were defined as foci-positive, and the percentage of positive nuclei was calculated as [(number of foci positive nuclei) / (number of nuclei scored)]* 100. A minimum of 500 nuclei per sample were scored.

\section{Generation of pDR-GFP expressing cells}


The CRC cells were seeded at $25 \times 10^{4}$ cells/well in 6-well plastic culture plates. The following day, cells were transfected with pDR-GFP plasmid (AddGene) using Lipofectamine 3000 following manufacturer's instruction. Seventy-two hours after infection, puromycin (Sigma Aldrich) was used to select stably infected cells.

\section{Plasmid based assay to measure homologous recombination}

The pDR-GFP-expressing cells were seeded at $25 \times 10^{4}$ cells/well in 6 -well plastic culture plates. The following day, cells were transfected with pCBASce-I expressing plasmid (AddGene) using Lipofectamine 3000 following manufacturer's instruction. Forty-eight to sixty hours after transfection, cells were harvested and analyzed by flow cytometry. The relative HR capacity was determined by dividing the percentage of GFP positive cells in Sce-I transfected cells by the basal percentage of GFP signal in mock control.

\section{Drug assays in organoids}

Organoids were enzymatically dissociated into single-cell suspensions using TrypLE ${ }^{\mathrm{TM}}$ Express Enzyme (ThermoFisher Scientific) for $15-30$ min at $37^{\circ} \mathrm{C}$. After washing, cells were counted and seeded in 2\% BME/growth media (5,000 - 6,000 cells/well) in 96-well plates pre-coated by BME (basement-membrane extract; Cultrex BME Type 2, Amsbio). Depending on the organoid sample, the drug treatment started between day two and ten after seeding when small organoid structures were visible. Cells were then treated with olaparib or oxaliplatin diluted in $2 \%$ BME/growth media for 18 days. Fresh $2 \%$ BME/organoid medium containing drugs was refreshed at least 3 times during the treatment. Organoid viability was assayed at the end of the experiment by CellTiter-Glo® (Promega) according to manufacturer's instruction with modifications. Briefly, reagent was mixed 1:1 with organoid media, and organoids were then subjected to the lysis by shaking at $600 \mathrm{rpm}$ for 15 minutes.

\section{In vivo treatment}

Established tumors were treated with the indicated drugs following specified schedule: olaparib: every day (from Monday to Friday), $50 \mathrm{mg} / \mathrm{kg}$ IP (vehicle: phosphate buffered saline (PBS) 10\% 2-hydroxy-propyl- $\beta$-cyclodextrin, Sigma); oxaliplatin: once weekly for a 
total of two treatments, $10 \mathrm{mg} / \mathrm{Kg}$ IP (vehicle: dextrose $5 \%$ water, Sigma-Aldrich). Tumor size was evaluated weekly by caliper measurements. Animal procedures were approved by the Ethical Commission of the Candiolo Cancer Institute and by the Italian Ministry of Health.

\section{Statistical Analysis}

Results were expressed as means \pm standard error of the mean (SEM) or standard deviation (SD) as indicated in the legend. Statistical significance was evaluated by $t$ test or two-way ANOVA using GraphPad Prism software. $p<0.05$ was considered statistically significant.

\section{RESULTS}

\section{A subset of CRC cells is sensitive to olaparib}

Metastatic CRCs bearing mutations in KRAS or BRAF and displaying stable microsatellite status (MSS) have dismal prognosis and are not eligible for therapies based on antiEGFR antibodies or immune-checkpoint blockade $(39,40)$. To model this subset, we selected ninety-nine CRC cell models enriched for KRAS and BRAF mutations or other alterations conferring resistance to EGFR blockade from our extensive collection (Supplementary Table S1). All CRC lines were challenged with five concentrations of olaparib $(0.5,1,5,10,15 \mu \mathrm{M})$. These concentrations were defined based on olaparib plasma levels known to be clinically achievable and therapeutically relevant for the treatment of ovarian and breast cancer patients $(41,42)$. The BRCA2 deficient pancreatic cell line (CAPAN1), which is sensitive to PARP blockade, served as positive control. Most of the tested CRC cell lines were markedly resistant across the entire range of olaparib concentrations. However, up to $13 \%$ of cell lines were sensitive at low drug concentrations at a level comparable to CAPAN1 cells (Fig. 1). Olaparib sensitive cell lines were also responsive to the other FDA-approved PARP inhibitors niraparib and rucaparib, underlying a likely drug class effect (Supplementary Fig. S1). When all three drugs were 
tested at equimolar concentrations, niraparib showed greatest potency, consistent with the previously reported higher PARP-trapping activity $(43,44)$.

\section{Genomic profiles and sensitivity to olaparib}

Mutations in KRAS, NRAS, or BRAF genes, which are commonly assessed as biomarkers in CRC, were not associated with sensitivity or resistance to olaparib (Fig. 1). None of the olaparib-sensitive cell lines were enriched for any of the transcriptional consensus molecular subtypes (CMS) of CRC (45). Nevertheless, none of olaparibsensitive cells classified as CMS2 (data not shown).

Considering that in breast and ovarian cancer sensitivity to PARP blockade is often associated with $B R C A 1$ and $B R C A 2$ mutations, we employed a clinically validated BRCA test (Cogentech, Milan, Italy). This analysis did not reveal mutations in either BRCA1 or $B R C A 2$ previously validated to be associated with HR deficiency and sensitivity to PARPi (Supplementary Table S2). Nevertheless, in silico assessment of a previously unreported BRCA2 variant in CX1 cells revealed the occurrence of a potentially pathogenic mutation, which has not yet been functionally assessed. In order to identify further molecular alterations underlying olaparib sensitivity, we performed WES analysis on the entire cell collection. This led to the identification of mutations in genes previously functionally associated to HR deficiency in human cells (Supplementary Table S3 and Supplementary Fig. S2).

HR deficiencies have been linked to distinct mutational signatures (46-48), which are combinations of nucleotide changes arising from specific mutagenesis processes, such as exposure to DNA damaging conditions and alteration in DNA replication processes (49). Over 30 mutational signatures have been identified in human cancers, a subset of which are linked to defective DNA repair pathways $(49,50)$. We therefore queried whether mutational signatures could discriminate olaparib-sensitive and resistant CRCs. The approach positively identified mutational profiles frequently occurring in $\mathrm{CRC}$, such as signatures 6 and 10 (Supplementary Fig. S2A). Of note, two out of 13 olaparib sensitive cells displayed signature number three which is correlated with HR defects and 
BRCAness $(47,49)$. Overall, however, mutational signatures did not discriminate between olaparib-sensitive and resistant CRCs (Supplementary Fig. S2A and S2B) $(51,52)$.

\section{Functional assays differentiate olaparib-sensitive and resistant CRC cells}

We reasoned that functional assays could be used to identify olaparib-sensitive colorectal cancer cells. To this end, we performed two types of analyses: first, we used radiation to induce DNA double strand breaks in olaparib sensitive and resistant models and measured the percentage of $\mathrm{\gamma H} 2 \mathrm{AX}$ and RAD51 foci positive nuclei post-radiation (Fig. $2 A)$. The percentage of $\mathrm{y} H 2 \mathrm{AX}$ foci positive nuclei increased comparably after radiation in both groups as expected (Fig. 2A, upper panel, Fig. 2B and Supplementary Fig. S3A). Notably, a marked difference between sensitive and resistant CRC models was observed in the RAD51 assay. Upon radiation, the percentage of RAD51 foci positive nuclei increased in all olaparib resistant cells, whereas the same effect was not observed in the sensitive cells (Fig. 2A, lower panel, Fig. 2C and Supplementary Fig. S3B).

Next, we directly measured HR capabilities using a two plasmid-system (pDR-GFP) designed to detect HR deficiencies in human cells (53). This strategy involves stable expression of two GFP cDNAs which are oriented as direct repeats and are separated by a drug selection marker. One of the GFP sequences is modified to contain the recognition site for the I-Scel endonuclease. Consequently, a double strand break (DSB) will be introduced in the GFP sequence when I-Scel is exogenously expressed (Fig. 3A). A homologous recombination event between the two GFP genes produces an intact GFP gene and expression of a functional GFP protein. The pDR-GFP plasmid was stably expressed in olaparib-sensitive and resistant cells (selected on the basis of their transfection efficiency) and the green fluorescent signal was measured by flow cytometry. GFP protein expression was observed in the olaparib-resistant cells (WiDr and DiFi) following I-Scel transfection, indicating that these cells were able to effectively repair the damaged GFP DNA. In contrast, olaparib-sensitive cells such as HROC278MET, KP363T and SKCO-1 were unable to repair the damaged DNA and therefore GFP expression was not detected following I-Scel transfection (Fig. 3B and Supplementary Fig. S4). 


\section{Cross-sensitivity between olaparib and oxaliplatin in CRC cells}

The results presented above highlight challenges in using genomic data to predict CRCs that are susceptible to PARP inhibition. We noted that in ovarian cancers the clinical development of the PARP inhibitors has encountered similar difficulties in using genomic correlates to identify patients likely to respond $(25,54)$. In ovarian cancer, sensitivity to platinum-based therapy is associated with subsequent clinical benefit from PARP inhibition and response to platinum is used as a criterion to select patients likely to respond to PARP blockade (25-27). We therefore hypothesized that CRCs sensitive to PARP blockade could also display cross sensitivity to oxaliplatin, a drug broadly used in combination regimens for treatment of bowel tumors (9). To test this possibility, we treated olaparib-sensitive and a subset of olaparib-resistant cells with a range of oxaliplatin concentrations (Fig. 4A, left and central panels). A significant correlation (Spearman $r=0.63, p=0.0005$,) between olaparib and oxaliplatin sensitivity was observed (Fig. 4B). To assess the specificity of this finding, we also tested sensitivity to 5-fluorouracil (5-FU, another widely used chemotherapeutic agent) in the same cell models and found no correlation (Fig. 4A, right panel and Fig. 4C).

\section{Olaparib and oxaliplatin sensitivity in patient-derived CRC organoids}

Considering that in our experience molecular correlates are unable to unequivocally predict sensitivity or resistance to olaparib in CRC, we wondered whether patient-derived CRC organoids (PDOs) could represent a functional platform to rapidly determine sensitivity to PARP blockade in this setting. We therefore analyzed five CRC organoids established either directly from tumor samples or from patient-derived xenografts (PDXs) (Supplementary Table S4). A PDO derived from a BRAF mutant PDX (patient \#1, case HROC278, Fig.5 A-C), from which we already derived olaparib-sensitive 2D cell lines (Fig. 1), displayed exquisite sensitivity to olaparib. Two organoids - derived from a NRAS mutant CRC specimen (patient \#2) or from an HER2 amplified PDX (patient \#3) - were also rather sensitive, although growth impairment could be appreciated only at higher concentrations of the PARP inhibitor. The remaining two models proved refractory across the entire range of olaparib concentrations (Fig. 5A-C). We next performed oxaliplatin 
tests on all five PDOs and found a striking cross sensitivity between olaparib and oxaliplatin (Fig. 5A-C).

To understand whether response to oxaliplatin treatment could be prospectively translated into prediction to olaparib response in patients, we collected clinical information on oxaliplatin-based treatment and response before tumor surgery and organoid generation in these five patients. While patient \#1 and \#5 did not receive oxaliplatin in the previous round of therapy, patients \#2, \#3 and \#4 were treated with oxaliplatin-containing regimens before surgery (Supplementary Table S4). Patient \#2 and patient \#3 achieved partial response $(P R)$ to previous oxaliplatin-based therapy and the corresponding organoids are consistently sensitive to oxaliplatin. Intriguingly, patient \#4 had derived clinical benefit from treatment with FOLFOX and panitumumab, but the organoids generated from his tumor were resistant to oxaliplatin. However, patient \#4-derived organoids were highly responsive to panitumumab (Supplementary Fig. S5), suggesting that clinical benefit in this patient could be associated to EGFR blockade rather than to oxaliplatin.

Overall, these results indicate that PDOs, which can be rapidly derived from surgical or bioptic CRC samples, can be effectively exploited to determine olaparib sensitivity. Furthermore cross-sensitivity between oxaliplatin and olaparib was maintained in patient derived models.

\section{Sequential olaparib and oxaliplatin treatment in patient-derived CRC xenografts}

We hypothesized that maintenance therapy with olaparib may be beneficial in CRCs that have experienced tumor shrinkage upon treatment with oxaliplatin based regimens. We reasoned that in vivo treatment of patient derived xenografts (PDXs) could help to ascertain whether olaparib and oxaliplatin could effectively be used sequentially in CRCs. This would have clinical relevance, for example when platinum toxicity becomes a limiting factor, a situation commonly experienced by CRC patients. To test this, we selected case HROC278 (carrying BRAF V600E), from which we derived 2D lines and organoids that are sensitive to olaparib (Fig.1 and Fig. 5). We had previously established two independent PDXs from this case, one from the primary tumor - HROC278 - and the other 
from a metastatic lesion - HROC278MET. When these PDXs were tested, we observed prolonged tumor stabilization in olaparib-treated mice compared with vehicle treated animals (Fig. 6A and B). Furthermore, we hypothesized that maintenance therapy with PARP blockade after initial oxaliplatin response could be effective in HROC278 (Fig. 6C); indeed, treatment with olaparib delayed progression after initial oxaliplatin-mediated tumor shrinkage (Fig. 6D).

\section{DISCUSSION}

In this work, we have assessed the anti-proliferative activity of PARP inhibition in a collection of ninety-nine CRC cell lines and found that up to $13 \%$ of them undergo growth arrest during two-week exposure of clinically achievable levels of olaparib. We note that the most sensitive $C R C$ lines were as responsive to olaparib as BRCA-deficient pancreatic or ovarian cancer lines (55). Although the primary screen was performed in two-dimensional tissue culture format, we also provide evidence that response to olaparib is maintained in clinically relevant models such as patient-derived organoid culture systems and xenograft models.

We elected to study microsatellite stable (MSS) cell lines which represent the patient population with the highest 'unmet clinical need score'; since MSI CRCs can be successfully treated with immunotherapy and, at least in the metastatic setting, are quite rare (56).

We initially explored whether biomarkers predictive of clinical benefit from PARP inhibitors in other malignancies could be applied to identify CRC models responsive to olaparib. A clinically approved BRCA1 and BRCA2 diagnostic test did not identify mutations in either $B R C A 1$ or $B R C A 2$ previously associated with HR deficiency and sensitivity to PARP inhibition. Exome sequencing was then employed to profile the status of HR genes. While mutation profiles of DNA repair genes could not distinguish between olaparib-sensitive and resistant lines, a relevant number of CRC lines carried one or more defect in genes involved in HR, leading to possible functional consequences. We acknowledge that we could not ascertain the germline or somatic nature of the identified variants likely to confer 
defective HR, since matched normal DNA was not available for most tumor cell lines. Importantly, the large amount of alterations in DNA damage response genes with unknown significance renders impracticable functional test for individual mutations and this approach was not performed in the present study.

Biallelic inactivation of $B R C A 1$ or $B R C A 2$ is associated with a pattern of genome-wide mutations known as signature 3, which reflects underlying deficient HR in breast cancer. Germline nonsense and frameshift variants in PALB2, as well as epigenetic silencing of $R A D 51 C$ and $B R C A 1$ by promoter methylation, can also give rise to the same signature in breast cancer and has been proposed as a biomarker to select tumors that may benefit from PARP inhibition (48). The term 'BRCAness' has been coined to describe tumors with features similar to those found in patients with germline BRCA1 or BRCA2 alterations, resulting in sensitivity to PARP inhibition and other DNA damaging agents due to defective HR (15). Although we positively identified mutational profiles signatures occurring in CRC such as numbers 6 and 10, only 2 out of 13 olaparib-sensitive cells displayed signature number 3 , which is correlated with HR defects and BRCAness $(47,49)$. In our experience, mutational signatures could not discriminate between olaparib sensitive and resistant CRCs.

In summary, our data suggest that genomic features associated with BRCAness or HR repair diagnostic assays (at least with current computational methods) do not entirely capture CRC tumors susceptible to PARP inhibition. On the contrary, we found that DNA repair functional tests are effective in discriminating sensitive and resistant CRC lines. Our results are concordant with previous studies in breast cancer organoid and PDX lines indicating that RAD51 nuclear foci are a surrogate marker of HR repair functionality $(57,58)$. Future studies should test the feasibility of detecting RAD51 in clinical CRC specimens.

We also found that sensitivity to oxaliplatin as determined in CRC models correlates with response to PARP blockade. In this regard, clinical development of the PARP inhibitors niraparib and rucaparib has been focused on platinum-sensitive ovarian cancer, thereby preferring clinical selection to molecular selection criteria. Sensitivity to platinum-based chemotherapy in ovarian cancer is associated with subsequent clinical benefit from PARP 
inhibition (21,25-27). Intriguingly, most of the CRC lines responsive to olaparib show cross-sensitivity to oxaliplatin, suggesting that oxaliplatin efficacy could be exploited to define 'clinical BRCAness'.

Importantly, we report that patient-derived organoids (PDOs) can be used to predict sensitivity to PARPi and that previous response to oxaliplatin-based regimens might predict for response to olaparib in CRC patients. It is therefore tempting to conclude that rapid establishment of organoids from clinical samples could in the future be effectively used in precision medicine programs aimed at selecting CRC patients likely to respond to PARP blockade.

Previous evidences in small datasets suggest that oxaliplatin could be efficacious in gastrointestinal tumors with HR defects such as pancreatic cancers $(23,59)$. Two recent case reports indicate that patients with BRCA1/2 mutant rectal tumors achieved a complete pathological response upon treatment with oxaliplatin containing neoadjuvant chemotherapy $(11,60)$. A phase II study of single agent olaparib in chemo-refractory metastatic colorectal cancer patients $(\mathrm{mCRC})$ was terminated prematurely due to lack of objective responses (30). A phase I study of olaparib in combination with irinotecan indicated increased toxicity and lack of clinical efficacy (61). However, these trials were conducted in a heavily pre-treated population of mixed MSS/MSI mCRC cases, without providing information about response to prior oxaliplatin treatment.

Our findings instead suggest that in mCRC PARP inhibition could be tested as maintenance therapy in the subset of patients characterized by underlying 'BRCAness' features and who achieved significant tumor shrinkage after FOLFOX-based induction first-line chemotherapy.

Since combinations of PARP inhibitors with oxaliplatin or irinotecan may be toxic (61), further opportunities for exploiting PARP inhibition in the clinic may come from combination with immune checkpoint inhibitors (https://clinicaltrials.gov ID: NCT03851614 and (62)).

Overall, the results presented in our work can be easily translated into clinical trials testing the efficacy of PARP inhibitors in $\mathrm{mCRC}$ patients who carry HR deficient tumors and have 
Author Manuscript Published OnlineFirst on December 12, 2019; DOI: 10.1158/1078-0432.CCR-19-2409

Author manuscripts have been peer reviewed and accepted for publication but have not yet been edited.

experienced profound tumor shrinkage upon induction first-line FOLFOX-chemotherapy. Contrary to the trials previously conducted in all comers, this therapeutic strategy is expected to improve progression-free survival and curb those quality-of- life-impairing side effects, such as neurotoxicity, associated to a prolonged oxaliplatin exposure.

Acknowledgments: The authors thank members of Molecular Oncology Laboratory at Candiolo Cancer Institute for critically reading the manuscript and, in particular, Gaia Grasso and Alberto Sogari for their help with experiments and constructive discussion, Giulia Della Chiara from INGM (Istituto Nazionale Genetica Molecolare "Romeo ed Enrica Invernizzi", Milan) for generating organoids from Patient \#2.

\section{References}

1. Van Cutsem E, Cervantes A, Adam R, Sobrero A, Van Krieken JH, Aderka D, et al. ESMO consensus guidelines for the management of patients with metastatic colorectal cancer. Ann Oncol 2016;27(8):1386-422 doi 10.1093/annonc/mdw235.

2. Overman MJ, Lonardi S, Wong KYM, Lenz HJ, Gelsomino F, Aglietta M, et al. Durable Clinical Benefit With Nivolumab Plus Ipilimumab in DNA Mismatch Repair-Deficient/Microsatellite Instability-High Metastatic Colorectal Cancer. J Clin Oncol 2018;36(8):773-9 doi 10.1200/JCO.2017.76.9901.

3. Le DT, Durham JN, Smith KN, Wang H, Bartlett BR, Aulakh LK, et al. Mismatch repair deficiency predicts response of solid tumors to PD-1 blockade. Science 2017;357(6349):409-13 doi 10.1126/science.aan6733.

4. Sartore-Bianchi A, Trusolino L, Martino C, Bencardino K, Lonardi S, Bergamo F, et al. Dualtargeted therapy with trastuzumab and lapatinib in treatment-refractory, KRAS codon 12/13 wild-type, HER2-positive metastatic colorectal cancer (HERACLES): a proof-of-concept, multicentre, open-label, phase 2 trial. Lancet Oncol 2016;17(6):738-46 doi 10.1016/S14702045(16)00150-9.

5. Van Cutsem E, Cuyle P-J, Huijberts S, Yaeger R, Schellens JHM, Elez E, et al. BEACON CRC study safety lead-in (SLI) in patients with BRAFV600E metastatic colorectal cancer (mCRC): Efficacy and tumor markers. J Clin Oncol 2018;36(4_suppl):627-doi 10.1200/JCO.2018.36.4_suppl.627.

6. Peeters M, Forget F, Karthaus M, Valladares-Ayerbes M, Zaniboni A, Demonty G, et al. Exploratory pooled analysis evaluating the effect of sequence of biological therapies on overall survival in patients with RAS wild-type metastatic colorectal carcinoma. ESMO Open 2018;3(2):e000297 doi 10.1136/esmoopen-2017-000297.

7. Cremolini C, Loupakis F, Antoniotti C, Lupi C, Sensi E, Lonardi S, et al. FOLFOXIRI plus bevacizumab versus FOLFIRI plus bevacizumab as first-line treatment of patients with metastatic colorectal cancer: updated overall survival and molecular subgroup analyses of the open-label, phase 3 TRIBE study. Lancet Oncol 2015;16(13):1306-15 doi 10.1016/S1470-2045(15)00122-9.

8. Tejpar S, Stintzing S, Ciardiello F, Tabernero J, Van Cutsem E, Beier F, et al. Prognostic and Predictive Relevance of Primary Tumor Location in Patients With RAS Wild-Type Metastatic Colorectal Cancer: Retrospective Analyses of the CRYSTAL and FIRE-3 Trials. JAMA Oncol 2016 doi 10.1001/jamaoncol.2016.3797. 
Author Manuscript Published OnlineFirst on December 12, 2019; DOI: 10.1158/1078-0432.CCR-19-2409

Author manuscripts have been peer reviewed and accepted for publication but have not yet been edited.

9. Lonardi S, Sobrero A, Rosati G, Di Bartolomeo M, Ronzoni M, Aprile G, et al. Phase III trial comparing 3-6 months of adjuvant FOLFOX4/XELOX in stage II-III colon cancer: safety and compliance in the TOSCA trial. Ann Oncol 2016;27(11):2074-81 doi 10.1093/annonc/mdw404.

10. Oh M, McBride A, Yun S, Bhattacharjee S, Slack M, Martin JR, et al. BRCA1 and BRCA2 Gene Mutations and Colorectal Cancer Risk: Systematic Review and Meta-analysis. J Natl Cancer Inst 2018;110(11):1178-89 doi 10.1093/jnci/djy148.

11. Soyano AE, Baldeo C, Kasi PM. BRCA Mutation and Its Association With Colorectal Cancer. Clin Colorectal Cancer 2018;17(4):e647-e50 doi 10.1016/j.clcc.2018.06.006.

12. AlDubayan SH, Giannakis M, Moore ND, Han GC, Reardon B, Hamada T, et al. Inherited DNARepair Defects in Colorectal Cancer. Am J Hum Genet 2018;102(3):401-14 doi 10.1016/j.ajhg.2018.01.018.

13. Heeke AL, Pishvaian MJ, Lynce F, Xiu J, Brody JR, Chen WJ, et al. Prevalence of Homologous Recombination-Related Gene Mutations Across Multiple Cancer Types. JCO Precis Oncol 2018;2018 doi 10.1200/PO.17.00286.

14. Knijnenburg TA, Wang L, Zimmermann MT, Chambwe N, Gao GF, Cherniack AD, et al. Genomic and Molecular Landscape of DNA Damage Repair Deficiency across The Cancer Genome Atlas. Cell Rep 2018;23(1):239-54 e6 doi 10.1016/j.celrep.2018.03.076.

15. Lord CJ, Ashworth A. BRCAness revisited. Nat Rev Cancer 2016;16(2):110-20 doi 10.1038/nrc.2015.21.

16. Bryant HE, Schultz N, Thomas HD, Parker KM, Flower D, Lopez E, et al. Specific killing of BRCA2deficient tumours with inhibitors of poly(ADP-ribose) polymerase. Nature 2005;434(7035):913-7 doi 10.1038/nature03443.

17. Tutt A, Tovey H, Cheang MCU, Kernaghan S, Kilburn L, Gazinska P, et al. Carboplatin in BRCA1/2mutated and triple-negative breast cancer BRCAness subgroups: the TNT Trial. Nat Med 2018;24(5):628-37 doi 10.1038/s41591-018-0009-7.

18. Kaufman B, Shapira-Frommer R, Schmutzler RK, Audeh MW, Friedlander M, Balmana J, et al. Olaparib monotherapy in patients with advanced cancer and a germline BRCA1/2 mutation. J Clin Oncol 2015;33(3):244-50 doi 10.1200/JCO.2014.56.2728.

19. de Bono J, Ramanathan RK, Mina L, Chugh R, Glaspy J, Rafii S, et al. Phase I, Dose-Escalation, Two-Part Trial of the PARP Inhibitor Talazoparib in Patients with Advanced Germline BRCA1/2 Mutations and Selected Sporadic Cancers. Cancer Discov 2017;7(6):620-9 doi 10.1158/21598290.CD-16-1250.

20. Litton JK, Rugo HS, Ettl J, Hurvitz SA, Goncalves A, Lee KH, et al. Talazoparib in Patients with Advanced Breast Cancer and a Germline BRCA Mutation. N Engl J Med 2018;379(8):753-63 doi 10.1056/NEJMoa1802905.

21. Moore K, Colombo N, Scambia G, Kim BG, Oaknin A, Friedlander M, et al. Maintenance Olaparib in Patients with Newly Diagnosed Advanced Ovarian Cancer. N Engl J Med 2018;379(26):2495505 doi 10.1056/NEJMoa1810858.

22. Mateo J, Carreira S, Sandhu S, Miranda S, Mossop H, Perez-Lopez R, et al. DNA-Repair Defects and Olaparib in Metastatic Prostate Cancer. N Engl J Med 2015;373(18):1697-708 doi 10.1056/NEJMoa1506859.

23. Golan T, Hammel P, Reni M, Van Cutsem E, Macarulla T, Hall MJ, et al. Maintenance Olaparib for Germline BRCA-Mutated Metastatic Pancreatic Cancer. N Engl J Med 2019 doi 10.1056/NEJMoa1903387.

24. Mateo J, Lord CJ, Serra V, Tutt A, Balmaña J, Castroviejo-Bermejo M, et al. A decade of clinical development of PARP inhibitors in perspective. Ann Oncol 2019 doi 10.1093/annonc/mdz192. 
Author Manuscript Published OnlineFirst on December 12, 2019; DOI: 10.1158/1078-0432.CCR-19-2409

Author manuscripts have been peer reviewed and accepted for publication but have not yet been edited.

25. Mirza MR, Monk BJ, Herrstedt J, Oza AM, Mahner S, Redondo A, et al. Niraparib Maintenance Therapy in Platinum-Sensitive, Recurrent Ovarian Cancer. N Engl J Med 2016;375(22):2154-64 doi 10.1056/NEJMoa1611310.

26. Ledermann JA, Harter P, Gourley C, Friedlander M, Vergote I, Rustin G, et al. Overall survival in patients with platinum-sensitive recurrent serous ovarian cancer receiving olaparib maintenance monotherapy: an updated analysis from a randomised, placebo-controlled, double-blind, phase 2 trial. Lancet Oncol 2016;17(11):1579-89 doi 10.1016/s1470-2045(16)30376-x.

27. Coleman RL, Oza AM, Lorusso D, Aghajanian C, Oaknin A, Dean A, et al. Rucaparib maintenance treatment for recurrent ovarian carcinoma after response to platinum therapy (ARIEL3): a randomised, double-blind, placebo-controlled, phase 3 trial. Lancet 2017;390(10106):1949-61 doi 10.1016/S0140-6736(17)32440-6.

28. Robson M, Im SA, Senkus E, Xu B, Domchek SM, Masuda N, et al. Olaparib for Metastatic Breast Cancer in Patients with a Germline BRCA Mutation. N Engl J Med 2017;377(6):523-33 doi 10.1056/NEJMoa1706450.

29. Pujade-Lauraine E, Ledermann JA, Selle F, Gebski V, Penson RT, Oza AM, et al. Olaparib tablets as maintenance therapy in patients with platinum-sensitive, relapsed ovarian cancer and a BRCA1/2 mutation (SOLO2/ENGOT-OV21): a double-blind, randomised, placebo-controlled, phase 3 trial. Lancet Oncol 2017;18(9):1274-84 doi 10.1016/S1470-2045(17)30469-2.

30. Leichman L, Groshen S, O'Neil BH, Messersmith W, Berlin J, Chan E, et al. Phase II Study of Olaparib (AZD-2281) After Standard Systemic Therapies for Disseminated Colorectal Cancer. Oncologist 2016;21(2):172-7 doi 10.1634/theoncologist.2015-0319.

31. Gorbunova V, Beck JT, Hofheinz RD, Garcia-Alfonso P, Nechaeva M, Cubillo Gracian A, et al. A phase 2 randomised study of veliparib plus FOLFIRI+/-bevacizumab versus placebo plus FOLFIRI+/-bevacizumab in metastatic colorectal cancer. Br J Cancer 2018 doi 10.1038/s41416018-0343-z.

32. Czito BG, Deming DA, Jameson GS, Mulcahy MF, Vaghefi H, Dudley MW, et al. Safety and tolerability of veliparib combined with capecitabine plus radiotherapy in patients with locally advanced rectal cancer: a phase $1 b$ study. Lancet Gastroenterol Hepatol 2017;2(6):418-26 doi 10.1016/S2468-1253(17)30012-2.

33. Pishvaian MJ, Slack RS, Jiang W, He AR, Hwang JJ, Hankin A, et al. A phase 2 study of the PARP inhibitor veliparib plus temozolomide in patients with heavily pretreated metastatic colorectal cancer. Cancer 2018;124(11):2337-46 doi 10.1002/cncr.31309.

34. Medico E, Russo M, Picco G, Cancelliere C, Valtorta E, Corti G, et al. The molecular landscape of colorectal cancer cell lines unveils clinically actionable kinase targets. Nat Commun 2015;6 doi 10.1038/ncomms8002.

35. Lazzari L, Corti G, Picco G, Isella C, Montone M, Arcella P, et al. Patient-Derived Xenografts and Matched Cell Lines Identify Pharmacogenomic Vulnerabilities in Colorectal Cancer. Clin Cancer Res 2019;25(20):6243-59 doi 10.1158/1078-0432.CCR-18-3440.

36. Corti G, Bartolini A, Crisafulli G, Novara L, Rospo G, Montone M, et al. A Genomic Analysis Workflow for Colorectal Cancer Precision Oncology. Clin Colorectal Cancer 2019;18(2):91-101.e3 doi 10.1016/j.clcc.2019.02.008.

37. Díaz-Gay M, Vila-Casadesús M, Franch-Expósito S, Hernández-Illán E, Lozano JJ, Castellví-Bel S. Mutational Signatures in Cancer (MuSiCa): a web application to implement mutational signatures analysis in cancer samples. BMC Bioinformatics 2018;19(1):224 doi 10.1186/s12859018-2234-y.

38. Arena S, Bellosillo B, Siravegna G, Martínez A, Cañadas I, Lazzari L, et al. Emergence of Multiple EGFR Extracellular Mutations during Cetuximab Treatment in Colorectal Cancer. Clin Cancer Res 2015 doi 10.1158/1078-0432.CCR-14-2821. 
Author Manuscript Published OnlineFirst on December 12, 2019; DOI: 10.1158/1078-0432.CCR-19-2409

Author manuscripts have been peer reviewed and accepted for publication but have not yet been edited.

39. Porru M, Pompili L, Caruso C, Biroccio A, Leonetti C. Targeting KRAS in metastatic colorectal cancer: current strategies and emerging opportunities. J Exp Clin Cancer Res 2018;37(1):57 doi 10.1186/s13046-018-0719-1.

40. Sanz-Garcia E, Argiles G, Elez E, Tabernero J. BRAF mutant colorectal cancer: prognosis, treatment, and new perspectives. Ann Oncol 2017;28(11):2648-57 doi 10.1093/annonc/mdx401.

41. Liston DR, Davis M. Clinically Relevant Concentrations of Anticancer Drugs: A Guide for Nonclinical Studies. Clin Cancer Res 2017;23(14):3489-98 doi 10.1158/1078-0432.CCR-16-3083.

42. de Haan R, Pluim D, van Triest B, van den Heuvel M, Peulen H, van Berlo D, et al. Improved pharmacodynamic (PD) assessment of low dose PARP inhibitor PD activity for radiotherapy and chemotherapy combination trials. Radiother Oncol 2018;126(3):443-9 doi 10.1016/j.radonc.2017.10.017.

43. Sun K, Mikule K, Wang Z, Poon G, Vaidyanathan A, Smith G, et al. A comparative pharmacokinetic study of PARP inhibitors demonstrates favorable properties for niraparib efficacy in preclinical tumor models. Oncotarget 2018;9(98):37080-96 doi 10.18632/oncotarget.26354.

44. Jiang X, Li W, Li X, Bai H, Zhang Z. Current status and future prospects of PARP inhibitor clinical trials in ovarian cancer. Cancer Manag Res 2019;11:4371-90 doi 10.2147/CMAR.S200524.

45. Guinney J, Dienstmann R, Wang X, de Reynies A, Schlicker A, Soneson C, et al. The consensus molecular subtypes of colorectal cancer. Nat Med 2015;21(11):1350-6 doi 10.1038/nm.3967.

46. Davies H, Glodzik D, Morganella S, Yates LR, Staaf J, Zou X, et al. HRDetect is a predictor of BRCA1 and BRCA2 deficiency based on mutational signatures. Nat Med 2017;23(4):517-25 doi $10.1038 / \mathrm{nm} .4292$.

47. Nik-Zainal S, Davies H, Staaf J, Ramakrishna M, Glodzik D, Zou X, et al. Landscape of somatic mutations in 560 breast cancer whole-genome sequences. Nature 2016;534(7605):47-54 doi 10.1038/nature17676.

48. Polak P, Kim J, Braunstein LZ, Karlic R, Haradhavala NJ, Tiao G, et al. A mutational signature reveals alterations underlying deficient homologous recombination repair in breast cancer. Nat Genet 2017;49(10):1476-86 doi 10.1038/ng.3934.

49. Alexandrov LB, Nik-Zainal S, Wedge DC, Aparicio SA, Behjati S, Biankin AV, et al. Signatures of mutational processes in human cancer. Nature 2013;500(7463):415-21 doi 10.1038/nature12477.

50. Petljak M, Alexandrov LB, Brammeld JS, Price S, Wedge DC, Grossmann S, et al. Characterizing Mutational Signatures in Human Cancer Cell Lines Reveals Episodic APOBEC Mutagenesis. Cell 2019;176(6):1282-94.e20 doi 10.1016/j.cell.2019.02.012.

51. Jiang X, Li X, Li W, Bai H, Zhang Z. PARP inhibitors in ovarian cancer: Sensitivity prediction and resistance mechanisms. J Cell Mol Med 2019;23(4):2303-13 doi 10.1111/jcmm.14133.

52. Hodgson DR, Dougherty BA, Lai Z, Fielding A, Grinsted L, Spencer S, et al. Candidate biomarkers of PARP inhibitor sensitivity in ovarian cancer beyond the BRCA genes. $\mathrm{Br} J$ Cancer 2018;119(11):1401-9 doi 10.1038/s41416-018-0274-8.

53. Pierce AJ, Johnson RD, Thompson LH, Jasin M. XRCC3 promotes homology-directed repair of DNA damage in mammalian cells. Genes Dev 1999;13(20):2633-8 doi 10.1101/gad.13.20.2633.

54. Swisher EM, Lin KK, Oza AM, Scott CL, Giordano H, Sun J, et al. Rucaparib in relapsed, platinumsensitive high-grade ovarian carcinoma (ARIEL2 Part 1): an international, multicentre, openlabel, phase 2 trial. Lancet Oncol 2017;18(1):75-87 doi 10.1016/S1470-2045(16)30559-9.

55. Stordal B, Timms K, Farrelly A, Gallagher D, Busschots S, Renaud M, et al. BRCA1/2 mutation analysis in 41 ovarian cell lines reveals only one functionally deleterious BRCA1 mutation. Mol Oncol 2013;7(3):567-79 doi 10.1016/j.molonc.2012.12.007. 
Author Manuscript Published OnlineFirst on December 12, 2019; DOI: 10.1158/1078-0432.CCR-19-2409

Author manuscripts have been peer reviewed and accepted for publication but have not yet been edited.

56. Venderbosch S, Nagtegaal ID, Maughan TS, Smith CG, Cheadle JP, Fisher D, et al. Mismatch Repair Status and BRAF Mutation Status in Metastatic Colorectal Cancer Patients: A Pooled Analysis of the CAIRO, CAIRO2, COIN, and FOCUS Studies. Clin Cancer Res 2014;20(20):5322-30 doi 10.1158/1078-0432.ccr-14-0332.

57. Castroviejo-Bermejo M, Cruz C, Llop-Guevara A, Gutierrez-Enriquez S, Ducy M, Ibrahim YH, et al. A RAD51 assay feasible in routine tumor samples calls PARP inhibitor response beyond BRCA mutation. EMBO Mol Med 2018;10(12) doi 10.15252/emmm.201809172.

58. Cruz C, Castroviejo-Bermejo M, Gutierrez-Enriquez S, Llop-Guevara A, Ibrahim YH, Gris-Oliver A, et al. RAD51 foci as a functional biomarker of homologous recombination repair and PARP inhibitor resistance in germline BRCA-mutated breast cancer. Annals of oncology : official journal of the European Society for Medical Oncology 2018;29(5):1203-10 doi 10.1093/annonc/mdy099.

59. Kondo T, Kanai M, Kou T, Sakuma T, Mochizuki H, Kamada M, et al. Association between homologous recombination repair gene mutations and response to oxaliplatin in pancreatic cancer. Oncotarget 2018;9(28):19817-25 doi 10.18632/oncotarget.24865.

60. Lin Z, Liu J, Peng L, Zhang D, Jin M, Wang J, et al. Complete pathological response following neoadjuvant FOLFOX chemotherapy in BRCA2-mutant locally advanced rectal cancer: a case report. BMC Cancer 2018;18(1):1253 doi 10.1186/s12885-018-5182-z.

61. Chen EX, Jonker DJ, Siu LL, McKeever K, Keller D, Wells J, et al. A Phase I study of olaparib and irinotecan in patients with colorectal cancer: Canadian Cancer Trials Group IND 187. Invest New Drugs 2016;34(4):450-7 doi 10.1007/s10637-016-0351-x.

62. Brown JS, Sundar R, Lopez J. Combining DNA damaging therapeutics with immunotherapy: more haste, less speed. Br J Cancer 2018;118(3):312-24 doi 10.1038/bjc.2017.376.

\section{Figure Legends}

Figure 1. A subset of $\mathrm{CRC}$ cells is sensitive to clinically relevant concentrations of the PARP inhibitor olaparib. Ninety-nine (99) CRC cells carrying alterations in RAS, BRAF or other genes conferring resistance to cetuximab (right panel and Supplementary Table S1) were tested for olaparib sensitivity by a long-term proliferation assay. $\mathrm{DiFi}$, a CRC cell line sensitive to cetuximab, and CAPAN1, a BRCA2-deficient pancreatic cell line sensitive to olaparib, were included as controls. Each cell line was tested at least twice with technical duplicates. Heatmap was plotted with Graphpad Prism software.

Figure 2. $\mathrm{Y}-\mathrm{H} 2 \mathrm{AX}$ and RAD51 profiles in olaparib resistant and sensitive cells upon ionizing radiation. A, Quantification of nuclear $\mathrm{Y}-\mathrm{H} 2 \mathrm{AX}$ foci (upper panel) and RAD51 foci (lower panel) in olaparib resistant and sensitive cells. Nuclei with five or more foci were scored as positive and at least 500 nuclei were counted for each sample. B, Immunofluorescence detection of DNA damage ( $\mathrm{Y}-\mathrm{H} 2 \mathrm{AX})$ and $(\mathbf{C})$ a marker of homologous recombination (RAD51) in olaparib resistant (COGA5) and sensitive (KP363T) cells treated as indicated. Four hours after irradiation, cells were fixed and stained. Nuclei are stained with DAPI (blue) and anti- $\gamma-\mathrm{H} 2 \mathrm{AX}$ antibody (red) or 
anti-RAD51 antibody (green). Scale bar: $50 \mu \mathrm{m}$. Representative images for two resistant and two sensitive cell lines are shown (see also Supplementary Fig. S3).

Figure 3. Homologous recombination assays in CRC cells sensitive and resistant to olaparib. A, Schematic representation of double-strand break (DSB) repair HR reporter assays. The pDR-GFP reporter plasmid contains a SceGFP gene, which includes an I-Scel site and inframe termination codons. An 812-bp internal GFP fragment (iGFP) can be used as a template to repair the DSB by HR-proficient cells which will generate a functional GFP gene whose green fluorescence can be detected by FACS. A puromycin selection gene is indicated in purple between the SceGFP and iGFP sequences. B, The indicated cells were initially transfected with the pDR-GFP plasmid. Next, stably expressing cells were transfected with the pCBASce-I to confer DNA damage and 50-60 hours after transfection cells were analyzed by flow cytometry. Quantification of HR capacity of each cell line relative to mock transfection (control) is reported in the bar graph. Results represent means \pm SD of at least two independent experiments. Statistical significance: ${ }^{* *} p<0.01$ (Student's t test). Ns indicates not statistically significant differences.

Figure 4. Sensitivity to olaparib correlates with oxaliplatin activity in CRC cell lines. A, A subgroup of twenty-six (26) cell lines initially screened with olaparib was tested with increasing concentrations of oxaliplatin $(0-12.5 \mu \mathrm{M})$ and 5-fluorouracil (5-FU, 0-20 $\mu \mathrm{M})$. B, Spearman analysis shows a significant correlation with oxaliplatin sensitivity $(p=0.0005)$, while no correlation was found with 5 -FU response $(\mathbf{C})$.

Figure 5. CRC patient-derived organoids shows cross-sensitivity to olaparib and oxaliplatin. A, Organoids derived from CRC patients or PDXs were disaggregated at single-cell level and plated on BME in 96-well plate. Once culture was established, treatment with olaparib or oxaliplatin at the indicated concentrations $(\mu \mathrm{M})$ was performed for 18 days. At the end of the experiment, organoids were microphotographed in bright field and representative pictures are shown. B, C, At the end of olaparib (B) or oxaliplatin (C) treatment, organoid viability was measured by CellTiter GLO assay. Results are average of two independent experiments with technical quadruplicates. Error bars represent SD.

Figure 6. Assessment of sensitivity to olaparib as single agent or as maintenance therapy after oxaliplatin treatment in patient-derived xenografts. A, B, PDXs obtained by two different lesions of the same patient, tumor (HROC278) (A) and metastasis (HROC278MET) (B), were tested for olaparib sensitivity $(50 \mathrm{mg} / \mathrm{kg})$ and treatment was started when tumors reached 200 $\mathrm{mm}^{3}$ (day 0 or black arrow). C, The PDX model obtained from HROC278MET was challenged with olaparib as maintenance therapy after initial oxaliplatin treatment. Oxaliplatin $(10 \mathrm{mg} / \mathrm{kg})$ was administered intraperitoneally once a week on days indicated by the dashed lines and olaparib treatment $(50 \mathrm{mg} / \mathrm{kg})$ was administered intraperitoneally starting after seven days from second oxaliplatin treatment (black arrow). D, Progression free survival of mice from experiment shown in panel C. A tumor volume $>500 \mathrm{~mm}^{3}$ was selected as an arbitrary endpoint. 
Figure 1

Author Manuscript Published OnlineFirst on December 12, 2019; DOI: 10.1158/1078-0432.CCR-19-2409

Author manuscripts have been peer reviewed and accepted for publication but have not yet been edited.

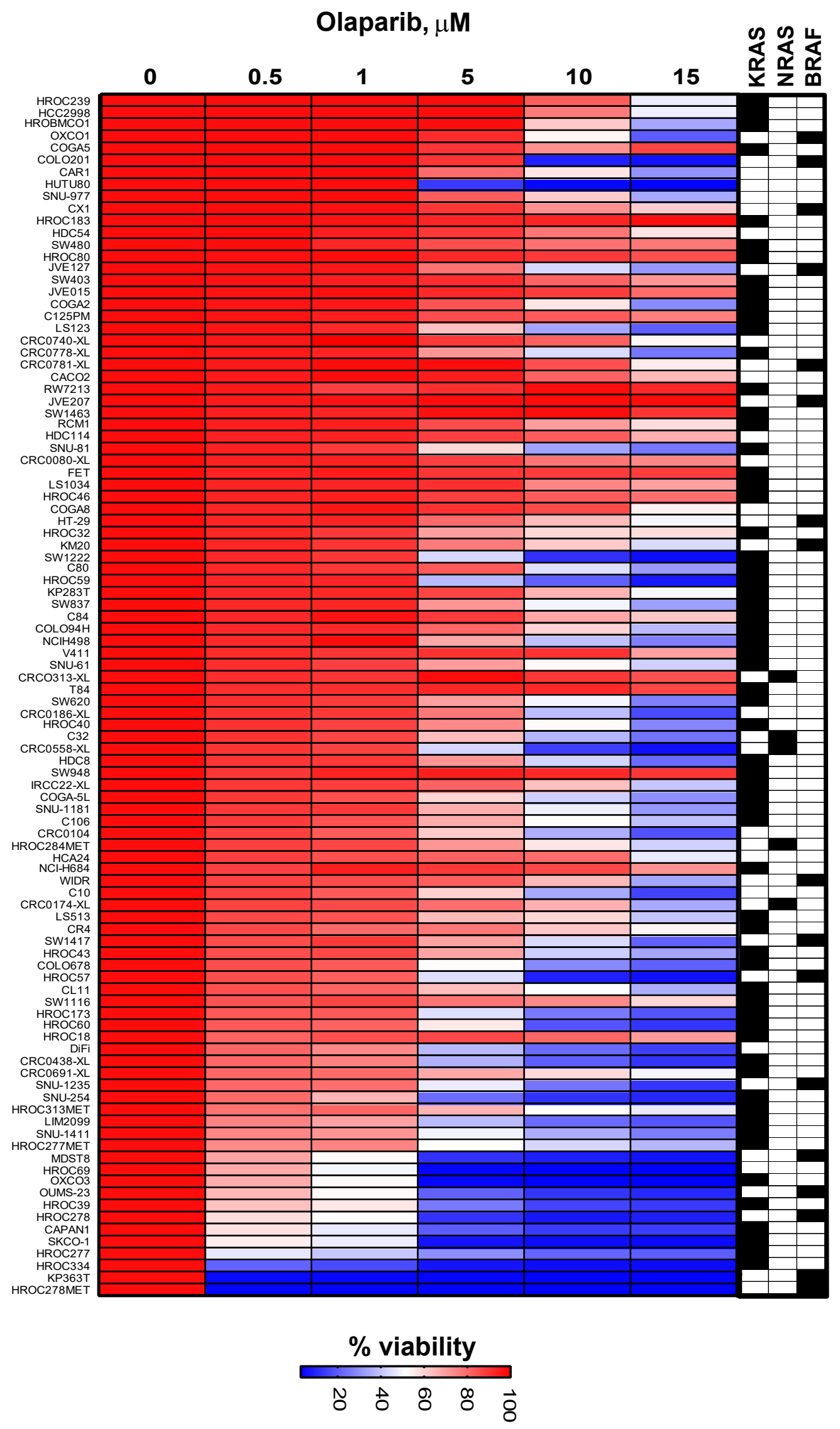


A Author Manuscript Published OnlineFirst on December 12, 2019; DOI: 10.1158/1078-0432.CCR-19-2409
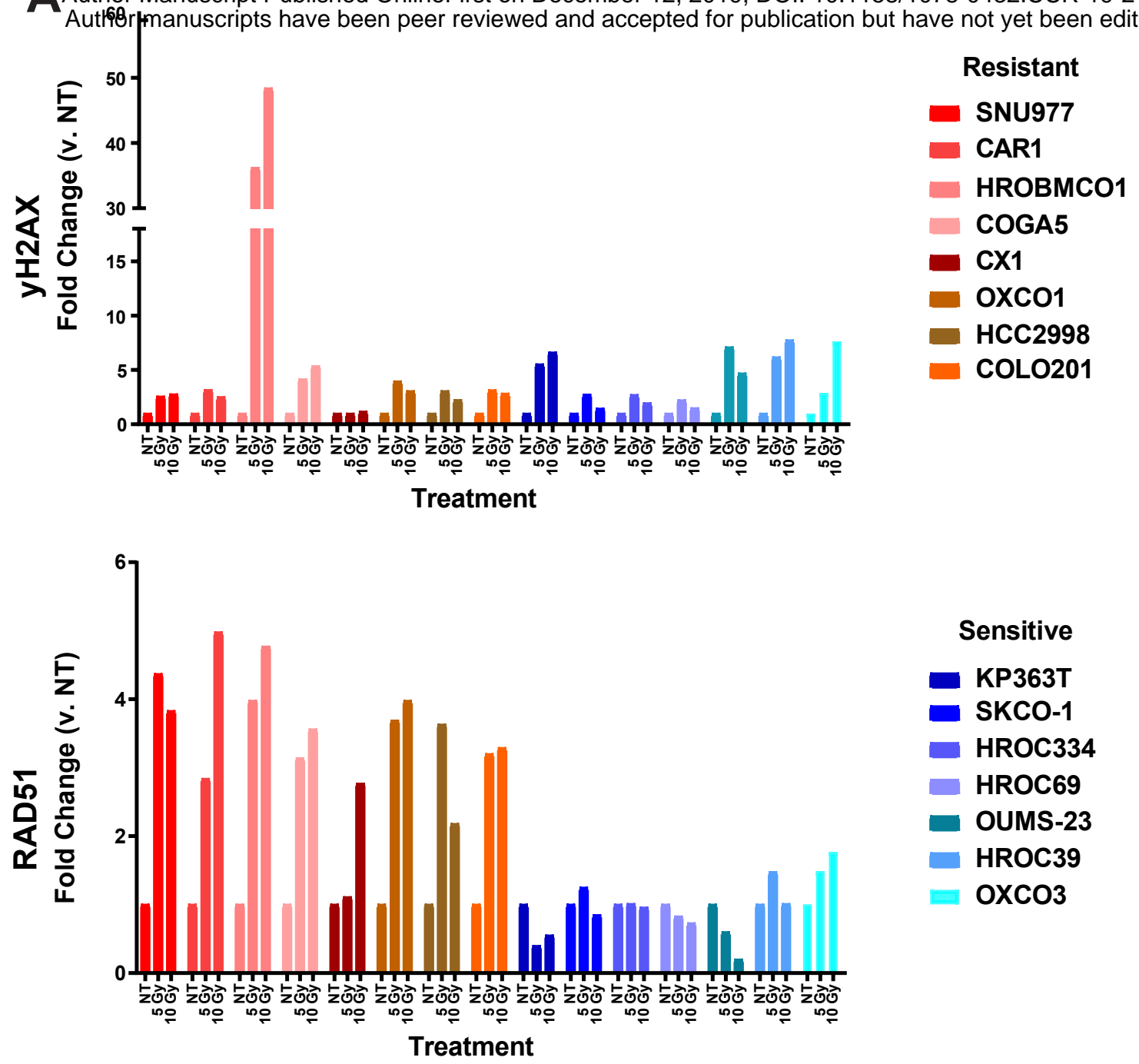

B
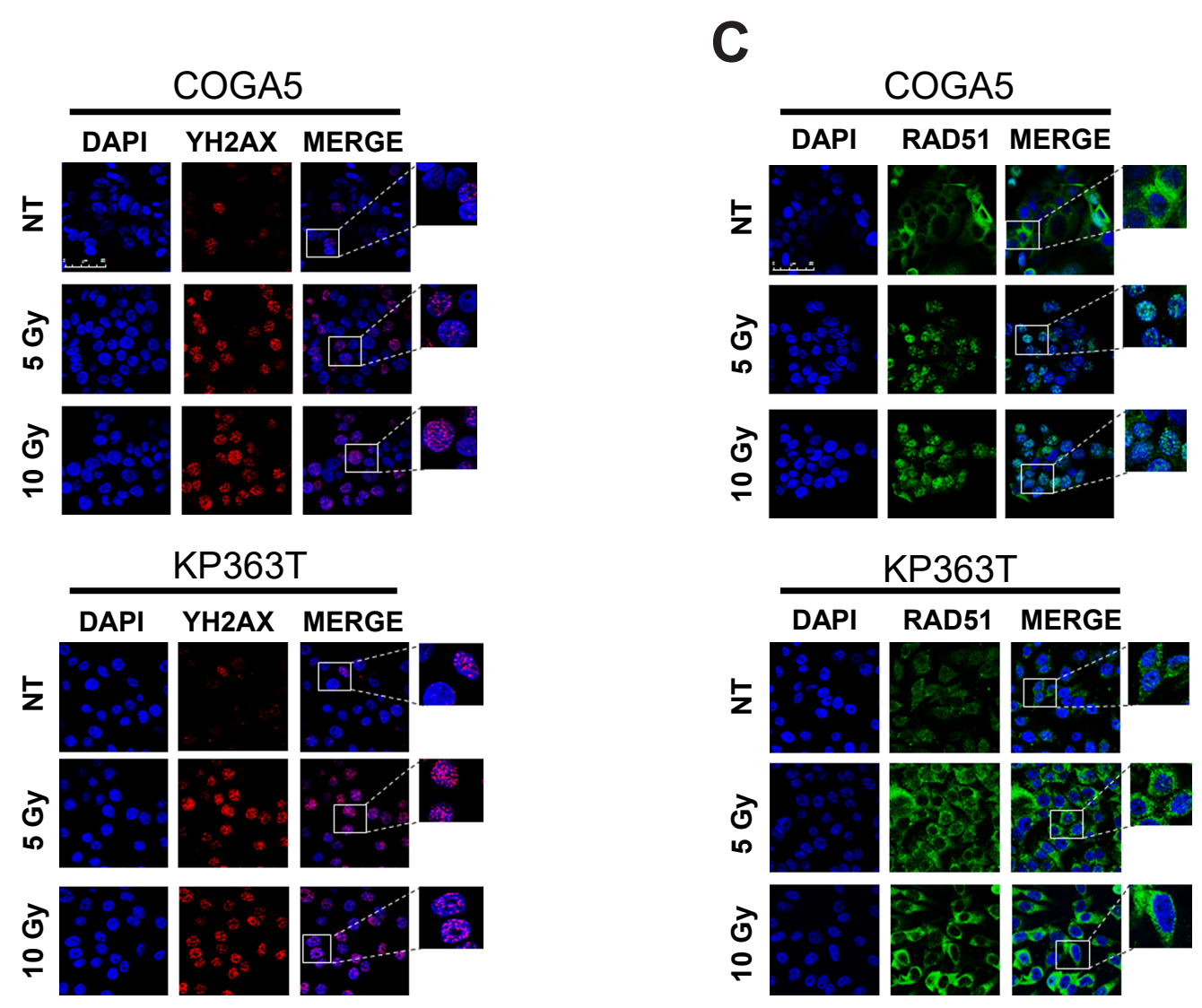
Figure 3

Author Manuscript Published OnlineFirst on December 12, 2019; DOI: 10.1158/1078-0432.CCR-19-2409 Author manuscripts have been peer reviewed and accepted for publication but have not yet been edited.

A

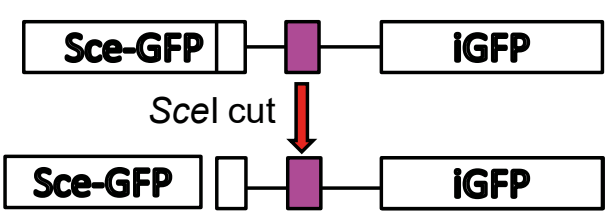

HR proficient cell $\$ HR NON-proficient cell

GFP

B

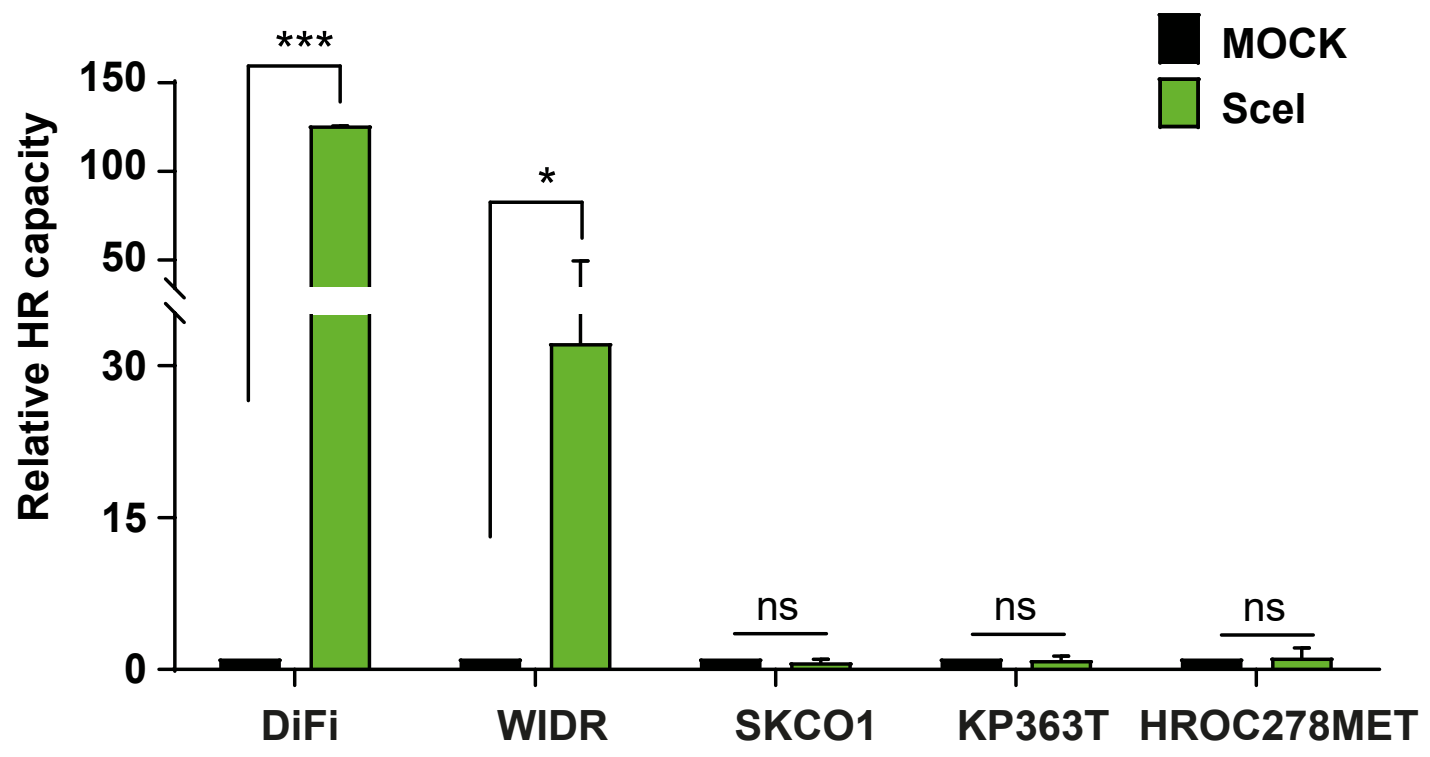


Author Manuscript Published OnlineFirst on December 12, 2019; DOI: 10.1158/1078-0432.CCR-19-2409 A
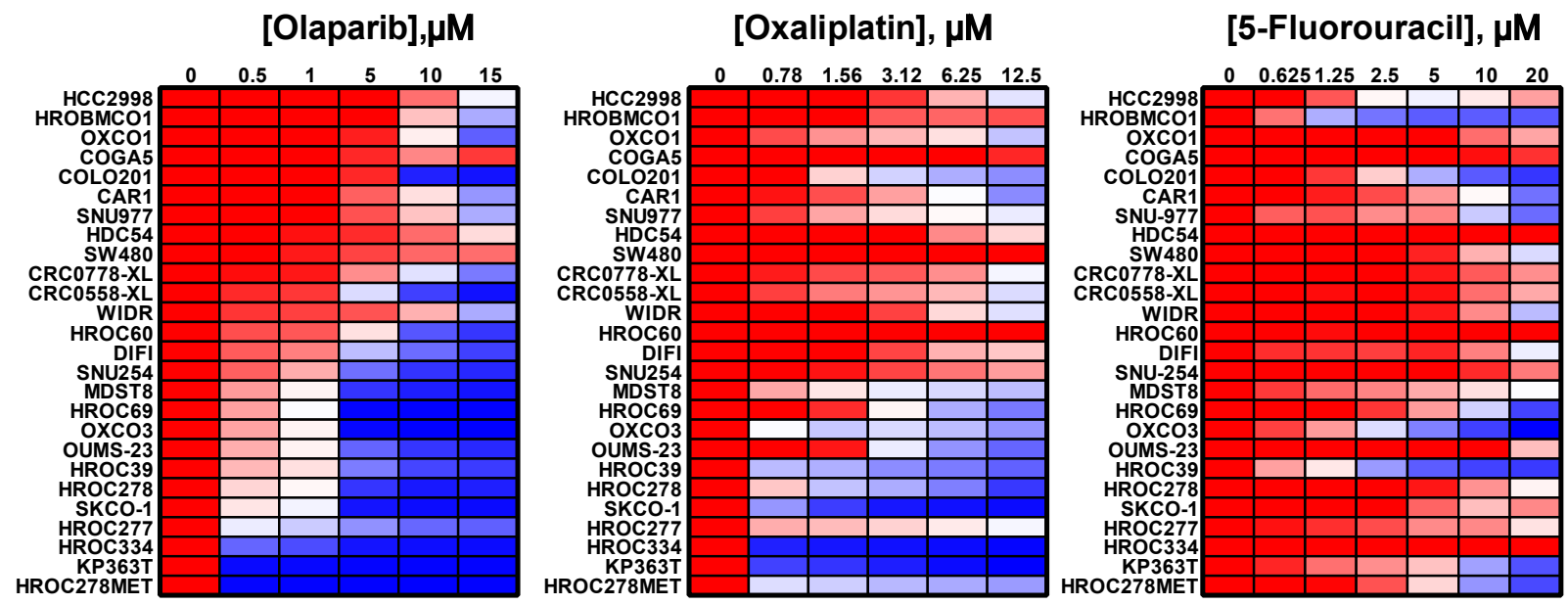

$\%$ viability

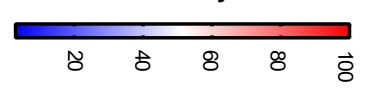

B

C
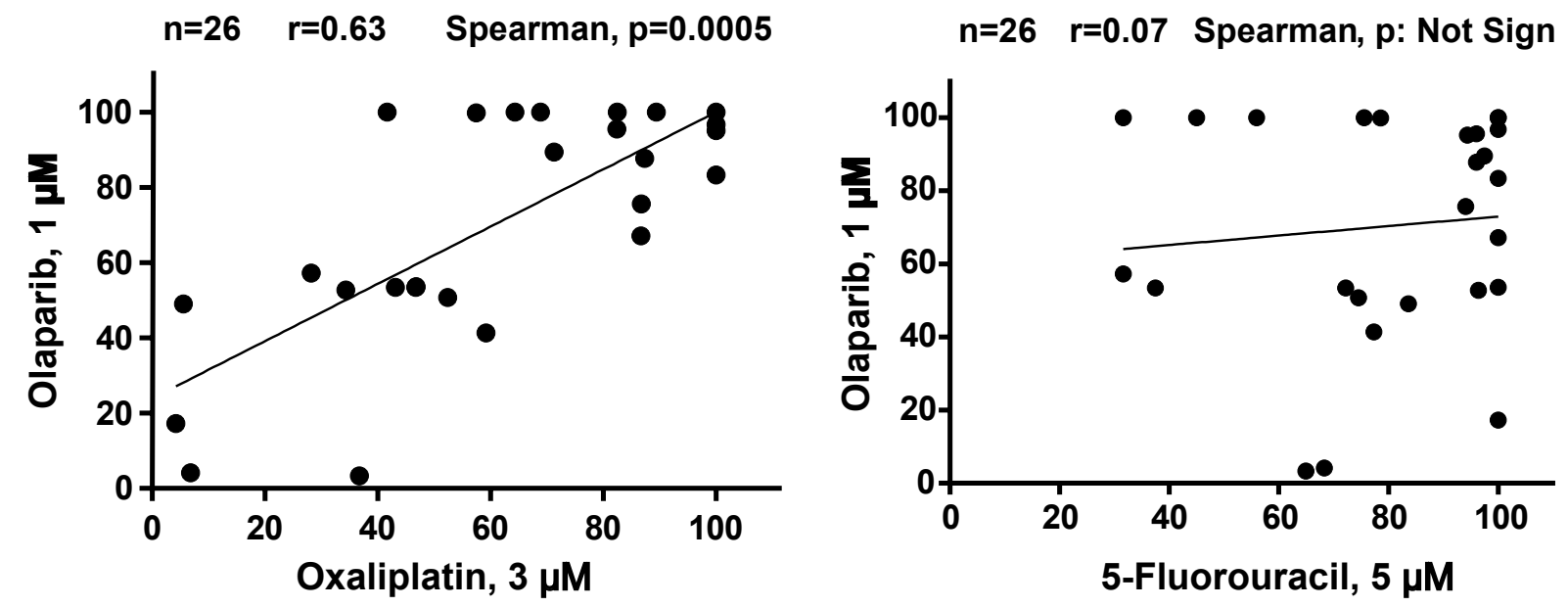


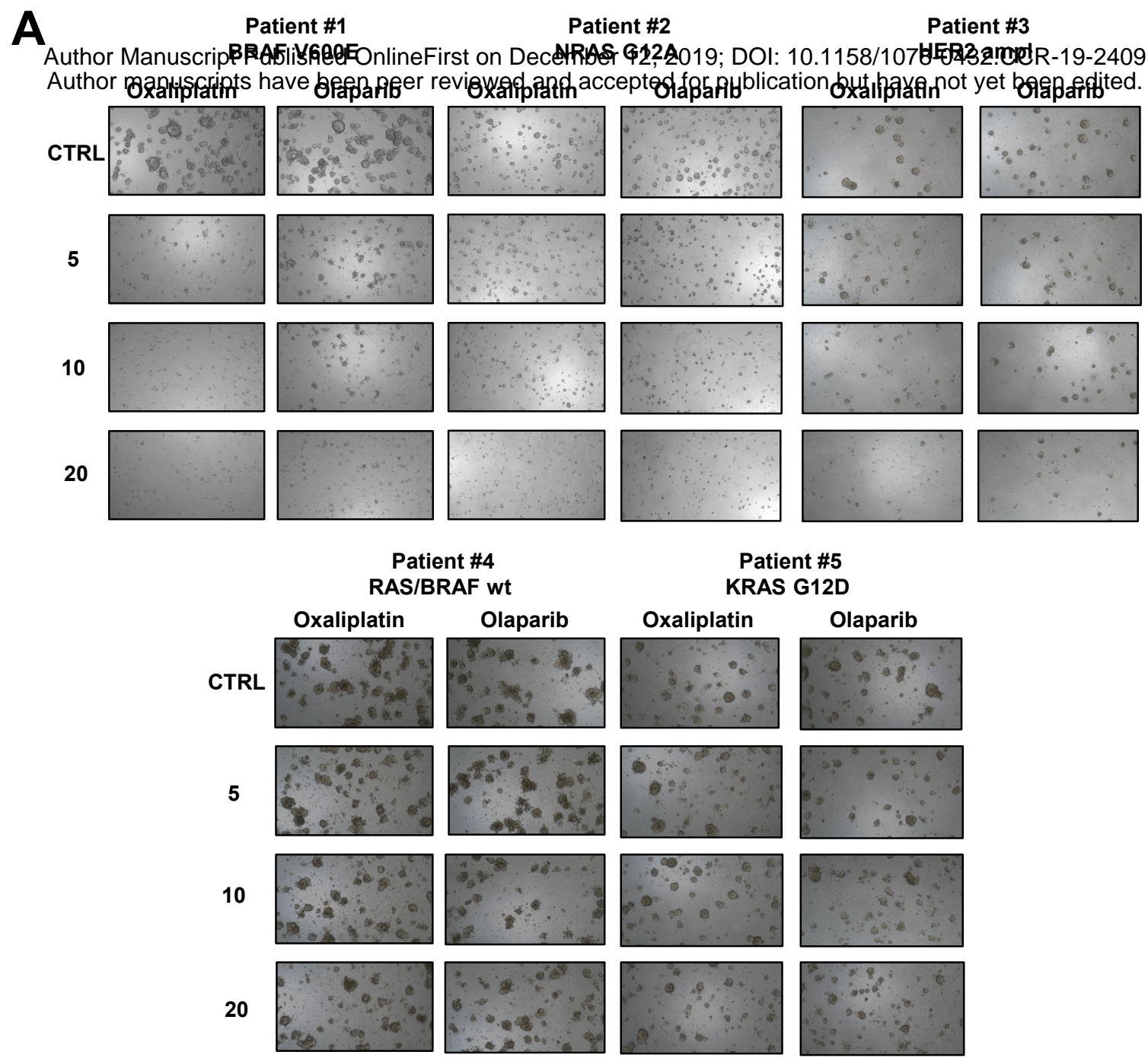

B

C

Olaparib

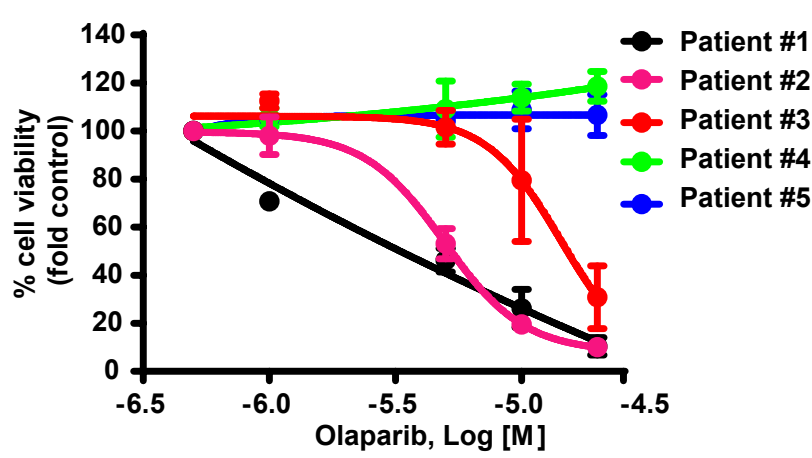

Oxaliplatin

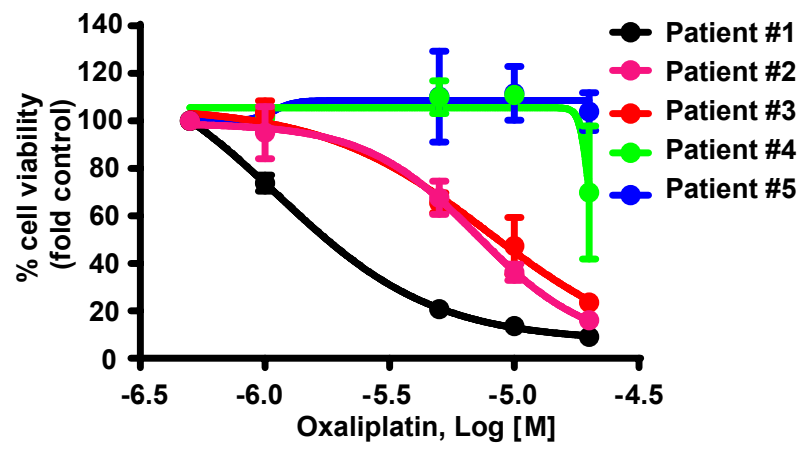


Author Manuscript Published OnlineFirst on December 12, 2019; DOI: 10.1158/1078-0432.CCR-19-2409 Author manuscripts have been peer reviewed and accepted for publication but have not yet been edited.

A

HROC278

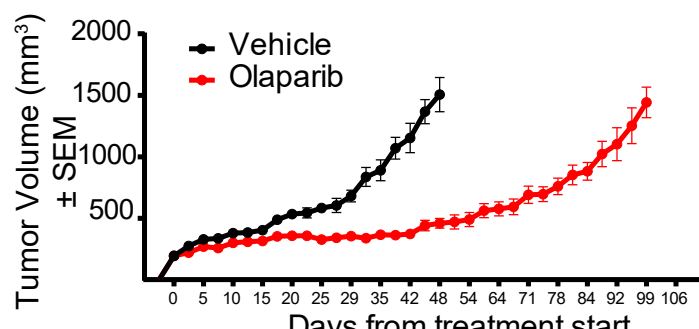

Days from treatment start
B

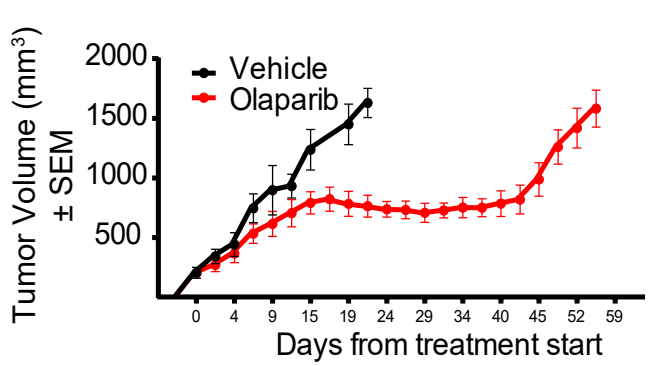

D

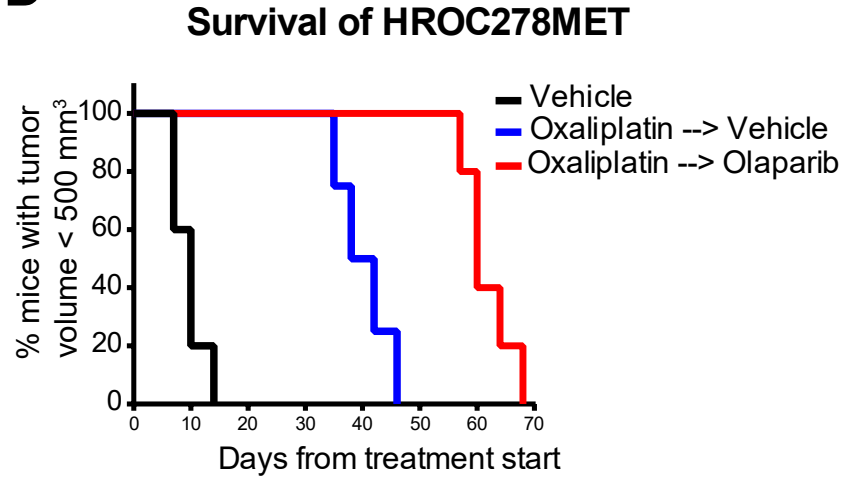


AACR

\section{Clinical Cancer Research}

\section{A subset of colorectal cancers with cross-sensitivity to olaparib and oxaliplatin}

Sabrina Arena, Giorgio Corti, Erika Durinikova, et al.

Clin Cancer Res Published OnlineFirst December 12, 2019.

Updated version Access the most recent version of this article at: doi:10.1158/1078-0432.CCR-19-2409

Supplementary Access the most recent supplemental material at:

Material http://clincancerres.aacrjournals.org/content/suppl/2019/12/12/1078-0432.CCR-19-2409.DC1

Author Author manuscripts have been peer reviewed and accepted for publication but have not yet been Manuscript edited.

E-mail alerts Sign up to receive free email-alerts related to this article or journal.

Reprints and To order reprints of this article or to subscribe to the journal, contact the AACR Publications Subscriptions Department at pubs@aacr.org.

Permissions To request permission to re-use all or part of this article, use this link http://clincancerres.aacrjournals.org/content/early/2019/12/12/1078-0432.CCR-19-2409. Click on "Request Permissions" which will take you to the Copyright Clearance Center's (CCC) Rightslink site. 